\title{
Evaluation of DECT for low Latency Real-time Industrial Control Networks
}

\author{
Kallol Das and Paul Havinga \\ Pervasive Systems Group, Faculty of EEMCS \\ University of Twente, Enschede, The Netherlands \\ Email: \{K.Das, P.J.M.Havinga $\} @$ utwente.nl
}

\begin{abstract}
Wireless sensor networks (WSNs) have revolutionized the industrial networks by enabling wireless sensing and control to the machine parts where wiring is impossible. However, new challenges in terms of communication reliability and latency, appear with the advances in the industrial wireless control systems. Existing standards are found inadequate to support many of these demanding situations as most of those are based on IEEE 802.15.4 standard, which is unable to provide high communication reliability with low latency (milliseconds). Digital Enhanced Cordless Telecommunications (DECT), a communication standard developed by European Telecommunications Standards Institute (ETSI), seems to support the timing and reliability requirements of modern industrial wireless control networks. In this paper, we evaluate the performance of DECT in various industrial environments and found that it can maintain excellent communication reliability between sensors and control centre with low latency in such scenarios.

Index Terms-Control systems, DECT, Real-time communication, Low latency, Reliability of communication, Industrial networks, Time varying shadow fading, Wireless sensor networks.
\end{abstract}

\section{INTRODUCTION}

Industrial networks can be divided into two major classes: (i) monitoring networks and (ii) control networks. Traditionally, both of these networks require extensive and costly wiring throughout the whole industry. Recent developments in wireless sensor networks (WSNs) help the industries to get rid of wires by enabling wireless communication between different machines and the control centre of the factory. This new technology is known as Machine-to-Machine (M2M) communication, which is rapidly becoming popular among the industrial experts [1], [2]. M2M systems not only reduce the set-up and running cost significantly but also enable the sensing of the machine parts where wiring is impossible (e.g., moving parts monitoring) [3]. Moreover, wireless M2M networks require less maintenance than that in wired networks. Inspired by these visions, some recent radio standards such as WirelessHART, ISA 100.11a have been developed to support industrial applications. These standards can satisfy the most of the requirements of industrial monitoring applications. However, different new challenges appear when researchers intend to use WSNs for industrial control systems, which were not visible in previously mentioned case. Such a study is conducted in [4], where the authors address the limitations of the existing radio technologies in industrial monitoring and control networks.

In wireless monitoring networks, the sensors are supposed to send information consists of few bytes to the base stations occasionally, i.e., the duty cycle of the radios used in these applications is very low. If a packet get delayed, no significant performance loss is observed. Even a few packet loss are tolerable in many applications. Hence, extremely reliable wireless communication is not generally required for such applications. If high delivery ratio is required for some monitoring applications, different techniques (e.g., retransmission scheme) can be applied to achieve it as long as delay in packet reception or low sensor duty cycling are accepted, such a protocol can be found in [5]. So, the main challenges of wireless monitoring applications are scalability and power consumption as thousands of sensor nodes might be engaged in data collection and they are expected to run for years on limited battery power.

On the other hand, control networks impose several challenges simultaneously. First of all, the sensors must maintain the connections with the control centre around the clock to guarantee the minimum delay in the control loop. This timing requirement (communication latency) may vary from few minutes to few milliseconds depending on the application. Applications like active vibration control in gas turbines used at the power stations impose low latency (milliseconds) real-time communication requirement [6]. Another issue is the reliability of the communication links. It might not be a problem if few packets are lost on the fly to make a control decision. However, it can be catastrophic if the packets containing control decisions are lost or get delayed in case of emergency. Last but not the least, high throughput often necessary in large control systems. An example is the 
case of vibration monitoring in which large amount of vibration data from different instruments has to be streamed for critical analysis. In this work, the goal is to identify a suitable radio technology and evaluate its performance for the scenarios where extremely reliable communications are essential along with high throughput and milliseconds level communication latency.

Naturally, the choice of radio technology should be from the widely used WSN standards such as WirelessHART and ISA 100.11a. Most of these standards are built on top of IEEE 802.15.4 standard, which operates in the unlicensed $2.4 \mathrm{GHz}$ band. As many other radio technologies such as WiFi, Blue-tooth, etc. also use this frequency band, the probability of packet collisions is quite high in this band. Moreover, the protocols do not support low communication latency, high throughput, and channel hopping to avoid interference in a dynamic environment. Eventually, reliable communication with milliseconds level communication latency cannot be guaranteed, which are key requirements in the control networks we are focusing at. ${ }^{1}$ Thus, WirelessHART and ISA 100.11a are not suitable for the delay critical control applications while providing satisfactory performance in monitoring and delay tolerant control applications. Clearly, the existing standards of wireless sensor communication cannot fulfil many requirements of the modern industrial control systems. Thus, we need new protocols that can cope with these situations.

The strict timing requirements of the control application we are targeting, seems achievable with a voice communication standard, namely DECT. In our previous work, we evaluated the performance of DECTULE in a dense WSN and found that it can maintain excellent communication performance with low latency in the network [7]. A brief performance parameter comparison between DECT and other widely used radio technologies in WSN can be found in the same work [7, see Table I]. To take this one step further, in this paper we evaluate DECT for low latency industrial control systems, where highly reliable communication links should be maintained with very tight timing.

We analyse the performance of DECT for control applications in office and various industrial environments with different sensor densities. The main contributions of this paper are as follows:

- We evaluate the quality of the channels used in sensor communications by calculating the signal to interference and noise ratio (SINR) under different conditions;

\footnotetext{
${ }^{1}$ Time Synchronized Mesh Protocol (TSMP) implemented in WirelessHART claims to achieve $99.9 \%$ reliability with several hundred milliseconds latency when the sensor duty cycle $(0.01 \%)$ is very low [5]. This does not fit with our requirements.
}

- We quantify the rate of lost connections in the network;

- We calculate the number of channel changes and the number of interfered connections in the network

- We propose a time varying industrial channel model and analyse performance of DECT control network in various shadow fading conditions.

The rest of the paper is organized as follows. In Section II, we briefly explain the features and operating principles of DECT. Section III introduces the simulation environment and formulates the problem with an explanation of a time varying shadow fading industrial channel model. The results are presented in Section IV, and finally we conclude this paper by outlining future research plans in Section V.

\section{FEATURES AND OPERATING PRINCIPLES OF DECT}

Before going into the details, we need to explain the features of DECT. DECT operates in the licensed frequency band of $1.88 \mathrm{GHz}$ to $1.9 \mathrm{GHz}$ in Europe. DECT can be considered as a hybrid system, which has two different types of devices in the network: the fixed parts (FPs)/infrastructure nodes and the portable parts (PPs)/mobile nodes. The FPs are generally wired between themselves to have centralized connections. A FP may have several radio fixed parts (RFPs). The 20 $\mathrm{MHz}$ band of DECT is divided among the 10 radio frequency carriers. Each of the carriers has 24 time slots in a $10 \mathrm{~ms}$ time frame. ${ }^{2}$ This creates 240 physical channels for communication on each time frame [7, see Figure 1]. However, a RFP/base station with single transceiver can only handle 12 simultaneous connections because the radio can not switch between frequencies in a particular time slot [8], [9]. DECT has relatively high throughput (1152 kbps) and long radio range (typically up to $75 \mathrm{~m}$ indoors and up to $300 \mathrm{~m}$ outdoors).

The operating principle of DECT is briefly explained below.

\section{A. Fixed part beacon and the portable locking proced- ure}

DECT network broadcasts beacons (service messages) to provide various information such as the access right, system information, paging information, etc. to the PPs. Every RFP broadcasts at least one beacon in each frame. In DECT, channel refers to a particular time slot(s) on a particular frequency carrier. At first, the RFPs select suitable channels to place the beacons. For this, DECT system follows the "listen before transmit"

\footnotetext{
${ }^{2} 12$ slots for down-link (communication from RFP to PP) followed by 12 up-link (communication from PP to RFP) slots.
} 
protocol. Being powered on for the first time, RFPs scan all the available physical channels to create a list of least interfered channels based on received signal strength indicator (RSSI). Then place their beacons on one of the suitable channels from the list, which are known as locked channels. The contents of the beacons are usually differs in frame to frame, as large amount of data containing all the system information are multiplexed into the beacon by the MAC layer [8]. DECT creates a multiframe by combining 16 frames to facilitates the MAC multiplexing. The RFPs change the channels for beacon broadcast in a regular interval even if there is no interference. The PPs have been notified about any change in locked channels in such case. This enables the PPs to switch the locked channel without loosing the synchronization.

When a PP wants to join in the network, it has to look for a beacon by listening to the RFPs on one of the locked channels. If several beacons have been received by a $P P$, it selects the beacon from the strongest RFP. The PP then checks the access right information to determine whether or not it has an access on that system. If accepted, several messages are exchanged to lock the PP into the system.

\section{B. Dynamic channel selection (DCS) and call set-up}

After being locked in the system, PPs can request for connection set-up to the RFPs any time. In DECT system, the connection set-up requests are known as calls. The unique feature of DECT standard is that the PPs initiate all the major communication protocols such as call set-up, handover (procedure of changing channel), etc. rather than FP. After powered on, the PPs are listening to the RFPs on the free channels to generate a list of suitable channels for communication. This list is updated periodically on idle mode (when the PPs do not have any data to send) and continuously during calls (active communication). Channels with high RSSIs are considered busy and the channels in which the PPs can't hear anything are the quietest channels. All the scanned channels are sorted into 12 levels with $6 \mathrm{~dB}$ interval based on the RSSIs [7, see Figure 2]. If possible, a PP selects a suitable channel from level 1 of the strongest RFP to set-up a call, otherwise it tries another channel in higher levels up to level 11 in a hierarchical order. In the next two frames, the PP checks the RSSI of that channel before finally selects it for call set-up. The PP sends a call request on that channel if the channel is free and the RSSI of the channel does not degrade significantly during these two frames. If the same channel is requested by another PP at the same time, there will be a collision and the PPs have to select different channels for the calls. This phenomena is known as channel selection failure [10]. The PPs can try another 5 suitable channels in the same RFP (if available) to set-up the call in case of channel selection failure. If still failed, the next two strongest RFPs (if available) can be tried using the same procedure. The call will be lost if no connection is made after trying 3 strongest RFPs [11].

\section{Paging}

Paging messages are used to inform a PP about an incoming call. FP sends these messages over the beacon throughout the network. Generally, this information is placed on the beacon of first frame (frame 0) of a multiframe. By doing this, the PPs only need to wake up once in every multiframe to check for a call, which help the PPs to save energy [9]. A connection is made only if the PP accepts the request, otherwise the call is abandoned.

\section{Call supervision and handover}

Any signal beyond the receiver sensitivity can be detected by a receiver. However, a minimum SINR should be maintained to decode a received signal successfully. All the PPs in DECT system monitor the RSSI and the SINR of every suitable channels during calls by using the free slots. If the PPs find another RFP with higher RSSI (at least $10 \mathrm{~dB}$ higher) than that of the present RFP, PPs request for handover. If no other suitable channels can be allocated immediately, the PPs continue to use the previous channels. Handover is also requested if PPs receive interference from other RFPs which are using same channel to communicate with other PPs. In this case, if the handover requests fail, the connections will be interfered and eventually be dropped. The only difference between a handover and a call set-up procedure is that a PP maintains two simultaneous connections during the handover, while one channel has been selected during a call set-up [11].

\section{PROBLEM FORMULATION}

\section{A. Network set-up}

In industrial control applications, the reliability of the wireless communication is more important than the number of sensors supported in the network. In this paper, we consider an industrial WSN where different machinery of a factory are wireless controlled from a control room. Sensors are attached to machines to gather different parameters such as vibration signatures, temperature readings, pressure, etc., while a actuators are deployed to control these machines according to the command of the control room. Suppose, DECT transceivers are attached to each pair of sensor-actuator to communicate wirelessly between the machines and the control room. In our network the control room 


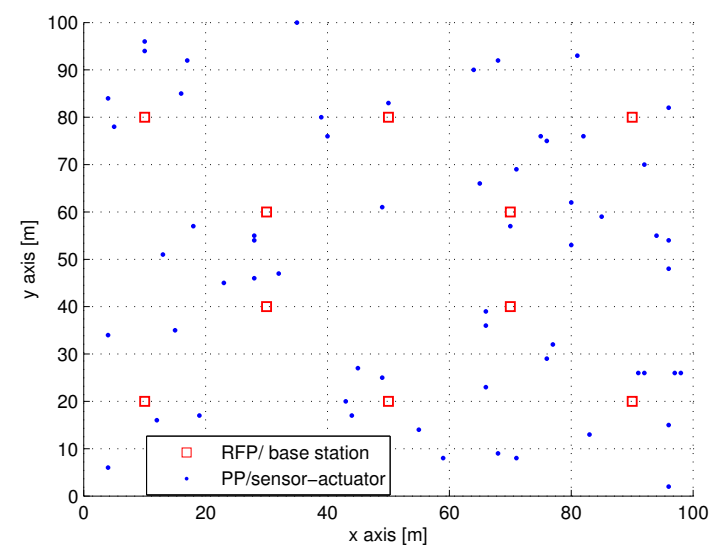

Figure 1. A DECT network for industrial control with 10 RFPs and 60 PPs.

collect sensor data through 10 RFPs, which are placed systematically in a $100 \mathrm{~m} \times 100 \mathrm{~m}$ factory and the machines are uniformly distributed in that area as shown in Figure 1. A number of PPs / sensor-actuator pairs (30-120) are attached to those machines. The RFPs are highly synchronized due to the wired connections, while the PPs get synchronize through the beacons. A total 50 random networks have been simulated for each set-up in MATLAB to generate the results.

\section{B. Traffic pattern}

Maximum 120 PPs (with 100\% duty cycle) are considered in our simulation because with 10 base stations we can keep at most 120 continuous connections in a DECT network. However, this doesn't limit the capability of DECT systems to support a large network with hundreds of PPs. The readers are refereed to [7], for results in such environment where DECT handles a dense network elegantly.

In DECT systems intended for voice communication, the call generation is usually modelled according to Poisson distribution. When a voice call has been initiated, it is expected to last for a while (typically, few minutes). The call duration in such networks is modelled as an exponential distribution by the authors in [12], [13]. On the other hand, in our industrial network, the intention is to monitor the machines continuously and control those with minimum delay if something happens. So, when a call has been established, it expects to last for a long time. As a result, the conventional models of call generation and call duration may not be suitable here.

We consider that the PPs will place the calls one after another in the beginning and suppose to maintain their connections for a long time. For our evaluation, we run the simulation for a long duration. However, for better visualization, we plot the results of first 2000 DECT frames for every PP. A certain number of reattempts for channel selection can take place if a suitable channel is not found, after that the call will be considered as lost. We do not attempt to re-establish a call if a PP loses its connection during any time after it established successfully.

\section{Propagation model}

Every radio signals suffer from free space path loss and shadowing during their travel from transmitter to receiver. These parameters depend on many things such as the geometry of the network, Doppler effect, obstacles etc. A correct propagation model is thus necessary to simulate a communication system. In this work, we use the ETSI propagation model of path loss, which can be represented as follows [14].

$$
L_{t}=L_{0}+10 \alpha \log (d)+K L_{f}+L_{s, t}+F,
$$

where, $L_{t}$ is the transmit power in $\mathrm{dBm}, L_{0}$ is the unit loss in $\mathrm{dB}$ (i.e., the loss after $1 \mathrm{~m}$ distance), $\alpha$ is the attenuation factor, $d$ is the distance between a PP and the respective RFP in $\mathrm{m}, K$ is the number of interposed floors, $L_{f}$ is the attenuation per floor in $\mathrm{dB}, L_{s}$ is the shadowing factor in $\mathrm{dB}, F$ is the multipath fading margin in $\mathrm{dB}$. To replicate an indoor office environment for our simulation, we set $L_{0}=30 \mathrm{~dB}, \alpha=3.5, K=1$, $L_{f}=15 \mathrm{~dB}$ and $F=10 \mathrm{~dB}$ (considering 99\% PPs have multipath with antenna diversity on).

The goal of this work is to evaluate DECT for harsh industrial environment. As a consequence, we have to modify the channel model accordingly. Generally, sensor-actuator pairs (i.e, the deployed PPs) are static in industrial environment, so no Doppler effect is considered in our simulation.

Typically, for indoor home/ office environment the shadowing effect is modelled as an uniformly distributed random number and the value of shadowing effect is considered as a constant for a transmitter-receiver pair during the whole call period. However, in factory environment, the movement of the machinery, highly reflective materials and electric impulses due to friction or other reasons can trigger time varying shadowing effect typically known as temporal fading. Tanghe et al. conducted a measurement campaign to model the large scale and temporal fading in the small industrial environment [15]. They modelled the large scale fading as a log-normal distribution and the temporal fading as a Rician distribution with mean $12.3 \mathrm{~dB}$ and standard deviation of $2.4 \mathrm{~dB} / \mathrm{sec}$ to $15.5 \mathrm{~dB} / \mathrm{sec}$. Similarly, we model the shadowing effects as a random variable over time for a particular transmitter-receiver pair to obtain industrial channel environment. For simplicity, we 
consider Gaussian distribution for time varying shadow fading. More explicitly, a random number is selected from a uniform distribution at first as proposed by ETSI for indoor DECT channels [14]. Then the selected random number is used as the mean of a Gaussian with a particular variance to generate the time varying nature of shadowing effects during a particular call. The value of this variance is varied from $(1 \mathrm{~dB} / 10 \mathrm{~ms})^{2}$ to $(3 \mathrm{~dB} / 10 \mathrm{~ms})^{2}$, to simulate a moderate to drastic shadowing effect of the industrial environment. ${ }^{3}$

Mathematically, $L_{s, t} \sim \mathcal{N}\left(\mu_{s}, \sigma^{2}\right) \mathrm{dB}$, where $\mu_{s}=\mathcal{U}(-10,10) \mathrm{dB}$. The industrial channel with $(1 \mathrm{~dB} / 10 \mathrm{~ms})^{2}$ shadow fading variance is considered as the reference for our simulation.

To set-up and maintain a call with acceptable bit error rate (BER) (typically, less than $10^{-3}$ ), a minimum SINR of $21 \mathrm{~dB}$ should be maintained in DECT [16], [17], [18]. During the SINR calculation, a transmission power of $24 \mathrm{dBm}$ and a background noise floor of $-100 \mathrm{dBm}$ are considered.

\section{EVALUATION RESULTS}

The communication quality and reliability of DECT systems largely depend on the DCS mechanism as this decides which channel to be used during a call. Therefore, to evaluate the performance of DECT in an industrial control network, we mainly have to evaluate the resource allocation method (i.e. DCS) of DECT. In our simulation, we evaluate the effect of node (traffic) density by varying the number of sensor-actuator pairs from low (30 PPs with 10 RFPs) to high (120 PPs, which is maximum allowable nodes with 10 RFPs) in the network. Note that all the PPs are always active (i.e., running with $100 \%$ duty cycling). As a performance checker, we analyse the communication latency, the number of handover, interfered calls, lost calls, SINR of the channels (used for calls), and average traffic per RFP in the network, which are explained below. Finally, we examine the effect of channel variation in Section IV-H by changing the time varying shadowing effects and also compare the results with office environment.

\section{A. Latency}

As mentioned earlier, DECT has a frame of $10 \mathrm{~ms}$ with TDD structure, when a slot is assigned on downlink for a connection after $5 \mathrm{~ms}$ another slot is allocated in uplink in the same time frame. In our network if a call is established and continued, a $10 \mathrm{~ms}$ communication latency is guaranteed. According to our simulation results in normal industrial environment, $10 \mathrm{~ms}$ latency can be guaranteed precisely (with maximum $0.1 \%$ outage)

\footnotetext{
${ }^{3} 10 \mathrm{~ms}$ comes from the DECT frame, i.e., in every frame during the call we have a sample for shadowing.
}

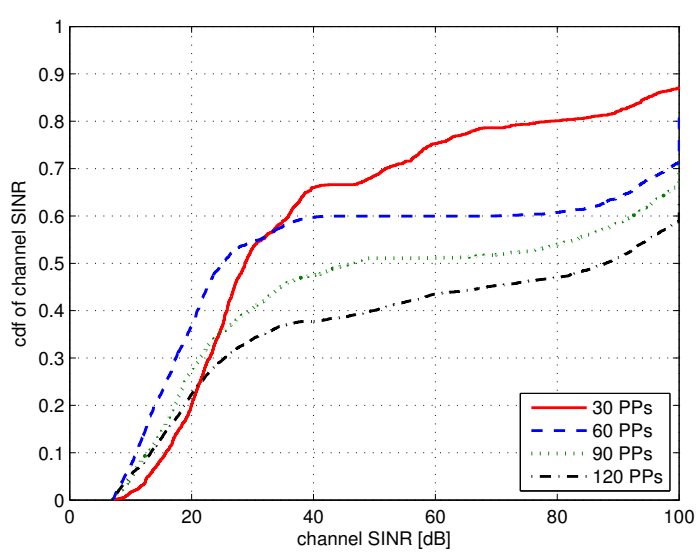

Figure 2. Channel quality comparison in different sensor densities.

for low-medium density networks (30-60 PPs with 10 RPFs) and with 1.49-7.63\% outage for high density networks (30-60 PPs with 10 RPFs). ${ }^{4}$ Communication latency analysis is more interesting in dense network environment, where the network is traffic modelled as a Possion distribution; such an analysis can be found in our previous work [7].

\section{B. Call handover}

The DCS mechanism of DECT elegantly handle the call supervision by changing the allocated channel during a call if necessary. A handover process may initiate due to two reasons. Firstly, if a PP finds another RFP with significantly higher RSSI and secondly, if the SINR of the presently used channel falls below the threshold of acceptable communication. During handover, a PP tries to select another suitable channel without releasing the old connection. A successful handover will only take place if the new selected channel fulfils all the quality requirements. The amount of handover attempts and successful handover in low sensor density are significantly low, while in high density case a huge amount of handover have been initiated and took place. In Table I, the number of successful handover attempts has been compared for different sensor-actuator pair (PP) densities.

\section{Interfered calls}

When an ongoing call experiences bad channel quality and the handover attempt (to find another suitable channel) is also failed, the call is interfered and eventually dropped. Ideally, this should not happen as the DCS takes care of it before the channel become too bad to maintain a connection. In our simulation, we

\footnotetext{
${ }^{4}$ Outage probability can be defined here as the probability of the PPs that can not achieve certain communication latency.
} 
get almost same number of interfered calls for every sensor-actuator pair density case (Table I). However, this number cannot represent any clear impression about the robustness of DECT in various cases. Along with the parameter lost calls (which is discussed in next subsection), interfered calls can clarify the capabilities of DECT in different sensor-actuator pair densities.

\section{Lost calls}

During call set-up, if a PP fails to select a suitable channel after trying all of its allowed recall attempts, the call is considered as a lost call. There are several reasons for this, which are as following, (i) more than one call requests on the same channel due to the back dated channel lists; (ii) poor channel SINR and (iii) PPs are in out of range of the RFPs. From Table I, it is clear that when the sensor-actuator pair density is low (3060 PPs), no calls are lost. For the high sensor-actuator pair density (90-120 PPs), the number of lost calls is significantly high. In this case, the number of handover initiation is also high. This means more channels are actually used by the PPs as a PP need to maintain two connections simultaneously during handover. As the PPs place the calls one after another, the actual number of free channels becomes smaller and smaller. Thus, the PPs which try to initiate their calls in the later phase, are unable to find free channels, and eventually those calls are lost.

\section{E. Channel quality}

The communication quality of any system can be quantified by the SINR of the channels used for communication. In DECT, a particular PP receives interference on a channel when the same channel has been used by other RFP-PP pairs in range. The SINR of a communication channel (which is used by a PP-RFP pair) may have different values in different frames during the call period, as the other RFP-PP pairs may or may not use the channel in question in every frames during that call period. In our simulation, we checked the SINR of every channels used for communication in every frames during the call period because a handover may take place if the SINR falls below the threshold. The complementary distribution function of the channel SINR in Figure 2 shows that more than $60 \%$ used channels are capable to maintain the minimum required SINR $(21 \mathrm{~dB})$ in all set-up. The channels, whose SINRs are below $21 \mathrm{~dB}$, actually trigger the handover. Unsuccessful handover attempts result in interfered call.

\section{F. Average traffic per RFP}

In Figure 3, the average load per RFP is shown for all the different sensor-actuator pair density cases. The average load per RFP is calculated by counting all the

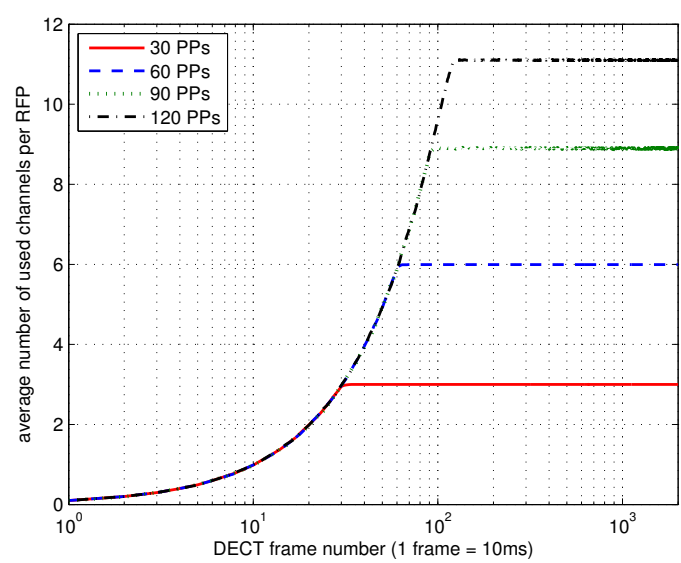

Figure 3. Average traffic comparison in different sensor densities.

used channels for communication by all the RFPs in a particular frame and taking the average among all the RFPs. In case of relatively low density (30-60 PPs) network, the average load per RFP is almost same as the maximum possible traffic, which is far below to the maximum allowed traffic per RFP. ${ }^{5}$ So, there are still enough rooms for new traffic in the network. In high density case, the average load per RFP is close to the maximum load capacity. In all the cases, the average traffic per base is increasing linearly with the frame number up to certain level because the PPs place the calls one after another and it becomes constant when all the PPs finished placing/ attempting for a call. It is clear from the Figure 3 that in case of the low PP density, the calls are in progress without any interruption (in most of the cases). However, in case of high density networks, some calls are interfered and lost. As a result, the average traffic in this case is slightly below the number of PPs per base ( 9 for 90 PPs and 12 for 120 PPs in the network with 10 RFPs). As, during handover process two channels are used for a call, the average traffic per base is slightly higher than the actual number of PPs on active calls.

The maximum traffic density considered in most DECT simulations for voice communication is about 5 Erlangs, which is also the suggested maximum traffic to design a network [14]. This can be considered as supporting 5 PPs continuously (with 100\% duty cycling) per base station. From our simulation, it is clear that the communication quality cannot be guaranteed in over crowded (with traffic more than 6 Erlangs) networks. However, we simulated the networks with traffics beyond the suggested limit to check the capacity of the networks.

\footnotetext{
${ }^{5}$ The maximum allowed traffic is 12 for a single RFP DECT system.
} 
Table I

COMPARISON ON NUMBER OF PLACED CALLS, LOST CALLS, INTERFERED CALL AND SUCCESSFUL HANDOVER BETWEEN NETWORKS WITH DIFFERENT SENSOR DENSITIES IN INDUSTRIAL ENVIRONMENT.

\begin{tabular}{|c|c|c|c|c|c|}
\hline $\begin{array}{c}\text { Sensor- } \\
\text { actuator } \\
\text { pair density }\end{array}$ & $\begin{array}{c}\text { Placed } \\
\text { calls }\end{array}$ & $\begin{array}{c}\text { Lost } \\
\text { calls }\end{array}$ & $\begin{array}{c}\text { Interfered } \\
\text { calls }\end{array}$ & $\begin{array}{c}\text { Lost and } \\
\text { interfered } \\
\text { calls }\end{array}$ & Handover \\
\hline 30 PPs & 1500 & 0 & 0 & 0 & 1 \\
\hline 60 PPs & 3000 & 0 & 3 & 3 & 14 \\
\hline 90 PPs & 4500 & 64 & 3 & 67 & 29580 \\
\hline 120 PPs & 6000 & 456 & 0 & 456 & 13121 \\
\hline
\end{tabular}

Table II

EFFECT OF CHANNEL VARIATION IN NETWORKS WITH DIFFERENT SENSOR DENSITIES.

\begin{tabular}{|c|c|c|c|c|c|}
\hline $\begin{array}{c}\text { Sensor- } \\
\text { actuator pair } \\
\text { density }\end{array}$ & $\begin{array}{c}\text { Placed } \\
\text { calls }\end{array}$ & $\begin{array}{c}\text { Lost } \\
\text { calls }\end{array}$ & $\begin{array}{c}\text { Interfered } \\
\text { calls }\end{array}$ & $\begin{array}{c}\text { Lost and } \\
\text { interfered } \\
\text { calls }\end{array}$ & Handover \\
\hline $\begin{array}{c}30 \text { PPs off } \\
\text { envt }\end{array}$ & 1500 & 0 & 0 & 0 & 1 \\
\hline $\begin{array}{c}30 \text { PPs ind } \\
\text { envt (std 1) }\end{array}$ & 1500 & 0 & 0 & 0 & 1 \\
\hline $\begin{array}{c}30 \text { PPs ind } \\
\text { ch 2 (std 2) }\end{array}$ & 1500 & 0 & 10 & 10 & 936 \\
\hline $\begin{array}{c}30 \text { PPs ind } \\
\text { ch 3 (std 3) }\end{array}$ & 1500 & 0 & 78 & 78 & 19300 \\
\hline $\begin{array}{c}60 \text { PPs off } \\
\text { envt }\end{array}$ & 3000 & 0 & 0 & 0 & 12 \\
\hline 60 PPs std 1 & 3000 & 0 & 3 & 3 & 14 \\
\hline $\begin{array}{c}60 \text { PPs ind } \\
\text { ch 2 (std 2) }\end{array}$ & 3000 & 1 & 21 & 22 & 1797 \\
\hline $\begin{array}{c}60 \text { PPs ind } \\
\text { ch 3 (std 3) }\end{array}$ & 3000 & 8 & 257 & 265 & 28541 \\
\hline
\end{tabular}

G. The overall performance comparison among different sensor density networks

While different performance matrices have been discussed in Sections IV-B to IV-F, Table I aggregates all the simulation results, which helps to compare the overall performance of different set-ups. From the lost and interfered calls column, it can be commented that the low sensor-actuator pair density networks can maintain excellent communication reliability, while significant disturbances are observed in high density networks. The number of handover requests increases with the PP density as the number of placed calls increases. This also increases the probability of collision. The only exception case is the 120 PPs in the network. In this case, the number of successful handover is lower than that in the network with 90 PPs. There are two reasons for this; firstly, many handover did not succeeded, which result in huge lost calls; secondly, as many PPs lost their connections the number of active PPs in the network becomes low as we did not attempt to re-establish the lost connections, which initiates relatively lower number of handover in the later phase.

\section{H. Effect of variation in channel model}

So far, we have evaluated the performance of DECT networks in a normal industrial environment where the channel parameters varies relatively slowly over time. As from the discussions above, we find that the network can only guarantee good communication quality for low to medium PP density cases, we will now examine the effect of channel variation only for those networks. We also observe the performance of the networks in office environment. To generate the channel in office environment, we removed the time varying shadowing from the propagation model. However, a uniformly distributed random shadowing is considered as proposed in ETSI model. The amount of this shadowing is considered as a constant for a RFP-PP pair during the whole call period. 30 PPs ind envt (std 1) represents the networks with $30 \mathrm{PPs}$, where a time varying industrial channel model with standard deviation $1 \mathrm{~dB} / 10 \mathrm{~ms}$ (according to in Equation 1) is used. Along with this normal industrial environment, we also considered two other industrial channels with fast shadow fading, where channel 2 represents a standard deviation of $2 \mathrm{~dB} / 10 \mathrm{~ms}$ and channel 3 represents a standard deviation of $3 \mathrm{~dB} / 10 \mathrm{~ms}$ in Equation 1. From the Lost and interfered calls column of Table II, we see that DECT works perfectly in the office environment (for which it was actually built) with no connection loss. It can still maintain satisfactory performance in normal industrial environments for both 30PPs and 60PPs in the network. The performance of the networks become poor if large variance in shadowing $\left((3 \mathrm{~dB} / 10 \mathrm{~ms})^{2}\right)$ has been applied. The number of handover attempts also increases significantly in those cases. Many PPs could not find single base station for a while in the high variance case, which is also a reason of the poor performance and huge amount of handover attempts.

In Figure 4, we show the quality of the channel (in terms of cdf of channel SINR) which are used for the connections. The quality of the channels is found to degrade with the increase in shadow fading variance. However, this figure does not give us the complete picture because in calculation of cdf, the SINRs of some channels are omitted, which are mentioned below. In high PP density cases, some of the PPs fail to select a suitable channel for the call which are considered as lost calls. The SINR calculation is not possible as no actual channels have been allocated for those calls. Again in case of large variance in shadowing effect, some of the calls are lost due to the channel unavailability. In this case, no RSSI readings are available on the PPs to calculate their SINRs. Thus, the cdf only represent the quality of the channels which are actually allocated for calls. From Table II, we also observe that the number 


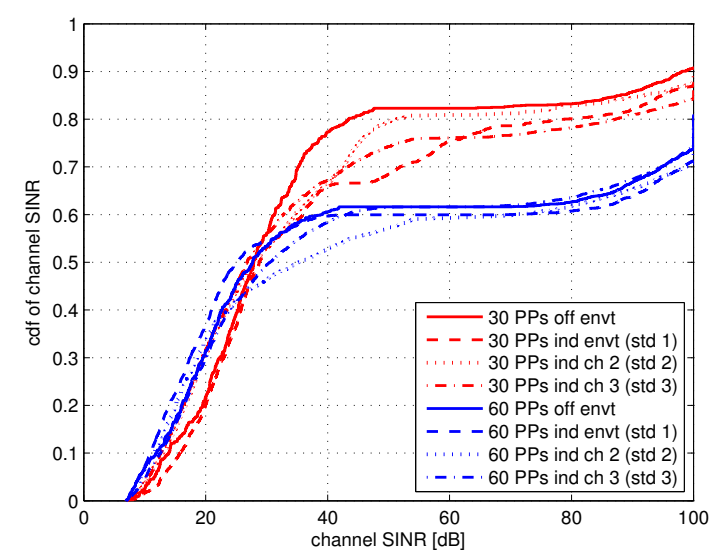

Figure 4. Comparison of channel quality in office and industrial environments.

of interfered calls is directly related to the number of handover requests.

In case of office environment, we found that 10 ms latency can be guaranteed with $100 \%$ reliability, while in industrial environments, we still can guarantee this for low-medium density networks with low outage probability $(0-0.73 \%)$. Only in extremely harsh industrial environment, we get high outage probability (5.2$8.83 \%$ ), however this type of fast time varying channel environment are rare.

\section{Conclusions}

Advances in WSNs demand the developments of new technologies for industrial control systems with increased capacity and reliability. This creates new challenges for the researchers to efficiently design the technologies. In this paper, we evaluate the performance of DECT in some of such envisioned industrial set-ups by analysing the communication reliability of DECT. Although, DECT was primarily developed for indoor cordless communications, the DCS mechanism of DECT makes it suitable for industrial networks. While providing excellent quality in office and relatively slow varying shadowing channel of industrial environments, DECT give poor performance in extremely fast shadow fading environment. However, the existence of such channels are quite unlikely compared to the channel variance measured by Tanghe et al.. Future works include design and implementation of robust wireless networking with DECT for low latency real-time industrial control systems, which can cope with the scenarios discussed in this paper and combat the multipath and fast shadow fading channels.

\section{ACKNOWLEDGEMENT}

This research is supported, in part, by the EU FP7ICT project WiBRATE (http://wibrate.eu), under the Grant No. 289041.

\section{REFERENCES}

[1] G. Lawton, "Machine-to-machine technology gears up for growth," Computer, vol. 37, no. 9, pp. 12 - 15, sept. 2004.

[2] K. Chang, A. Soong, M. Tseng, and Z. Xiang, "Global wireless machine-to-machine standardization," IEEE Internet Computing, vol. 15, no. 2, pp. $64-69$, mar-apr 2011.

[3] H.-J. Korber, H. Wattar, and G. Scholl, "Modular wireless realtime sensor/actuator network for factory automation applications," IEEE Trans. on Industrial Informatics, vol. 3, no. 2, pp. $111-119$, may 2007.

[4] P. Zand, S. Chatterjea, K. Das, and P. Havinga, "Wireless industrial monitoring and control networks: The journey so far and the road ahead," J. Sens. Actuator Netw., vol. 1, no. 2, pp. 123-152, 2012.

[5] K. Pister and L. Doherty, "TSMP: Time synchronized mesh protocol," IASTED Distributed Sens. Netw., pp. 391-398, 2008.

[6] S. Tewani, B. Walcott, and K. Rouch, "Active optimal vibration control using dynamic absorber," in Proc. of IEEE Int. Conf. on Robotics and Automation, vol. 2, apr 1991, pp. 1182 -1187.

[7] K. Das and P. Havinga, "Evaluation of DECT-ULE for robust communication in dense wireless sensor networks," in Proc. of $3 r d$ Int. Conf. on the Internet of Things (IoT 2012), China, Oct. 2012.

[8] Digital Enhanced Cordless Telecommunications (DECT), Common Interface (CI); Part 1-5, ETSI Std. v2.3.1, 2010.

[9] J. Phillips and G. Mac Namee, Personal wireless communication with DECT and PWT. Artech House, 1998.

[10] A. Capone and L. Musumeci, "A fast dynamic channel assignment algorithm for packet services in DECT," ACM SIGMOBILE Mobile Computing and Communications Review, vol. 1, no. 2, pp. 25-32, Jul 1997.

[11] H. Eriksson and R. Bownds, "Performance of dynamic channel allocation in the DECT system," in Proc. of the 41st IEEE Vehicular Tech. Conf., may 1991, pp. $693-698$.

[12] S. McCann and A. Croft, "High density DECT system simulation performance," in Proc. of IEE Colloquium on CT2/CAI and DECT Cordless Telecommunications, nov 1990, pp. 9/1 -9/4.

[13] R. Atkinson and J. Dunlop, "Comparison of ISMA and DECTDCS channel access policies for indoor wireless communications systems," Electronics Letters, vol. 36, no. 15, pp. 1307 -1309, jul 2000.

[14] "Radio Equipment and Systems (RES); Digital European Cordless Telecommunications (DECT): A Guide to DECT features that influence the traffic capacity and the maintenance of high radio link transmission quality, including the results of simulations," ETSI, Tech. Rep. ETR 042, July 1992.

[15] E. Tanghe, W. Joseph, L. Verloock, L. Martens, H. Capoen, $\mathrm{K}$. Van Herwegen, and W. Vantomme, "The industrial indoor channel: large-scale and temporal fading at 900, 2400, and 5200 mhz," IEEE Trans. on Wireless Comm., vol. 7, no. 7, pp. 2740 -2751 , jul 2008.

[16] J. Punt, D. Sparreboom, F. Brouwer, and R. Prasad, "Mathematical analysis of dynamic channel selection in indoor mobile wireless communication systems," IEEE Trans. on Vehicular Technology, vol. 47, no. 4, pp. 1302 -1313, nov 1998.

[17] J. Casademont, J. Paradells, and M. Lopez Carrillo, "Power control in the DECT radio local loop," in Proc. of IEEE 6th Int. Conf. on Universal Personal Comm., vol. 2, oct 1997, pp. $785-789$ vol.2.

[18] V. Degli-Esposti, G. Falciasecca, M. Frullone, and G. Riva, "Influence of propagation model accuracy on DECT system performance assessments," in Proc. of 26th European Microwave Conf., vol. 2, sep 1996, pp. 920 -924. 